\title{
Menos desejabilidade social é mais desejável: Neutralização de instrumentos avaliativos de personalidade
}

\author{
Ariela Raissa Lima Costa \\ Nelson Hauck Filho
}

\section{RESUMO}

Respostas socialmente desejáveis (RSD) acontecem quando alguém faz uma autoavaliação excessivamente positiva de si mesmo em instrumentos psicométricos de autorrelato. A presença de itens com conteúdo valorativo (desejável ou indesejável) é uma das causas das RSD, uma vez que o indivíduo pode responder motivado mais pela popularidade do conteúdo e menos pelo traço descritivo de fato. 0 objetivo do estudo foi elaborar uma versão menos socialmente desejável de dois instrumentos avaliativos de traços de personalidade, o Big Five Inventory e o IPIP-Modesty Scale-A5. A amostra foi de 379 universitários, de 18 a 47 anos (M = 22,53 anos; $D P=6,2$ ). Os participantes responderam às versões originais e neutralizadas dos instrumentos e a um instrumento que avalia desejabilidade social, o IPIP - Impression Management and Self Deception Scales. Pôde-se observar que as versões neutralizadas se correlacionaram positiva e fortemente com suas versões originais, e menos com o instrumento de desejabilidade social. A estrutura fatorial das versões neutralizadas também foi bastante similar àquela dos instrumentos originais. Os achados evidenciam a eficiência do procedimento de neutralização no controle da desejabilidade social.

Palavras-chave: desejabilidade social; modéstia; cinco grandes fatores; construção de escalas.

\section{ABSTRACT}

\section{Less social desirability is more desirable: Neutralizing personality inventories}

Socially desirable responding (SDR) occurs when someone does an overly positive assessment about him or herself using a self-report inventory. The existence of evaluative content (desirable or undesirable) in self-report items is regarded as one of the causes of SDR, as an individual might rate items motivated more by content popularity and less by the descriptive trait. The aim of this study was to elaborate a less socially desirable version of two self-report inventories of personality traits, the Big Five Inventory and the IPIP Modesty Scale-A5. Participants were 379 university students, from 18 to 47 years $(M=22.53$ years; $S D=6.2)$, who responded to the original and to a neutralized version of each instrument, as well as to the IPIP Impression Management and Self Deception Scales. As expected, results revealed that our neutralized instruments were positively and strongly correlated to their original versions, besides less correlated to social desirability. Factor structure of both original instruments was replicated in their neutralized versions. Findings attest to the feasibility and efficiency of the neutralization procedure in the controlling of social desirability.

Keywords: social desirability; humility; big five factors; test construction.

As pessoas são, em geral, motivadas pela aprovação social, o que interfere na maneira como interagem umas com as outras. Naturalmente, essa preocupação com a aparência pode se estender para situações de testagem psicológica, principalmente quando a personalidade é o foco da avaliação. Em alguns casos, o testando pode estar interessado em demonstrar que possui algumas características valorizadas socialmente, assim endossando itens que sugerem que ele é, por exemplo, sociável, criativo, dinâmico, confiável ou resiliente, mesmo que não possua todas essas características. A essa exacerbação das qualidades com o objetivo de ge-

\section{Sobre os Autores}

A.R.L.C.

orcid.org/0000-0002-5942-6466

Universidade São Francisco

Campinas, SP

arielalima10@gmail.com

N.H.F.

orcid.org/0000-0003-0121-7079

Universidade São Francisco -

Campinas, SP

hauck.nf@gmail.com

\section{Direitos Autorais}

Este é um artigo de acesso aberto e pode ser reproduzido livremente, distribuído,

transmitido ou modificado, por qualquer pessoa desde que usado sem fins comerciais. $O$ trabalho é disponibilizado sob a licença Creative Commons CCBY-NC.

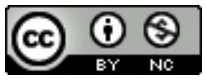




\section{INTERACÃo EM 4. PSICOLOGIA}

rar uma imagem específica de si mesmo para o avaliador é dado o nome de resposta socialmente desejável (RSD) (PauIhus, 1991). Desejabilidade social, por sua vez, designa o valor atribuído a um conjunto de atitudes ou comportamentos pelos indivíduos membros de um dado contexto social. As RSD são um fenômeno complexo, que ocorrem por uma variedade de motivos. Alguns dos mais importantes são o resultado esperado do teste, o contexto em que a avaliação ocorre, as características pessoais do examinando e a escrita dos itens específicos (Ziegler, 2015). Embora os três primeiros elementos sejam mais difíceis de serem controlados, o último deles - a escrita dos itens - possibilita modificações e aprimoramentos visando à redução de RSD, o que é o foco desta pesquisa. No presente trabalho, são elaboradas versões alternativas de dois instrumentos de autorrelato que avaliam personalidade, buscando diminuir a probabilidade de RSD por meio do método de neutralização valorativa de itens, desenvolvido por Bäckström (2007).

A avaliação psicométrica via autorrelato possui diversas vantagens. Entre elas, vale mencionar o fato de possibilitar, ao próprio sujeito, expressar comportamentos e vivências internas, além de reduzir o tempo necessário para tal avaliação, e não exigir treinamento especializado na aplicação do teste (Paulhus \& Vazire, 2007). Um item de autorrelato costuma descrever comportamentos, sentimentos e pensamentos, solicitando à pessoa que pontue o quanto o conteúdo tem a ver com ela. No entanto, instrumentos de autorrelato são também, potencialmente, suscetíveis a vieses de resposta. Por exemplo, algumas das situações descritas nos itens são percebidas como mais socialmente aceitas, enquanto outras podem ser percebidas como "tabus", o que pode influenciar no quanto o item será endossado pelas pessoas. Em outras palavras, além do conteúdo descritivo do item, que se refere ao traço de interesse, em geral está presente uma segunda dimensão, conhecida como "conteúdo valorativo". Esse segundo tipo de conteúdo se refere a quão "bom" ou "ruim", de acordo com o contexto cultural, é visto alguém com o traço descrito pelo item. Peabody (1967) demonstrou, empiricamente, que pares de descritores como audacioso (bold) e imprudente (rash), ou ainda cauteloso (cautious) e retraído (timid), são equivalentes de um ponto de vista descritivo, mas opostos em termos de desejabilidade social. Baseado na análise fatorial de um conjunto de quádruplas de indicadores como esses, o autor encontrou uma fundamentação empírica para a separação entre conteúdo descritivo e valorativo de itens, chamando atenção para a importância do assunto.

Respostas socialmente desejáveis (RSD) ocorrem enquanto expressão de duas dimensões latentes do funcionamento cognitivo (Paulhus \& Reid, 1991). A primeira delas é chamada de manejo de impressão e consiste em uma tendência a oferecer, deliberadamente, respostas distorcidas com a intenção de causar uma impressão específica. Por exemplo, um examinando pode antecipar que a vaga de emprego para a qual está se candidatando requer que o funcionário seja carismático, o que pode motivá-lo a tentar passar a impressão, nos testes psicológicos, de que possui essa característica. A segunda dimensão, chamada de autoengano, consiste em uma tendência inconsciente a manifestar crenças irrealisticamente positivas acerca de si mesmo (Paulhus, 2002). Por exemplo, alguém pode ver a si mesmo como dono de uma bondade sobre-humana (mesmo que isso contradiga a percepção de todos os informantes familiarizados com essa pessoa), e responder aos itens do teste de acordo com essa autopercepção enviesada.

Existe uma conexão bastante direta entre a presença de conteúdo valorativo em um item de autorrelato e a ocorrência de RSD a esse item específico. Se o enunciado de um item contém palavras com valência negativa ou positiva muito intensas, esses estímulos podem atuar como um gatilho, ativando processos relacionados a uma ou mais das dimensões das RSD, promovendo então distorções nas respostas aos itens (consciente ou inconscientemente, dependendo do caso). Assim, itens que usam palavras com valência afetiva muito positiva, isto é, que apresentem conteúdos mais explicitamente desejáveis, serão mais fáceis de serem manipulados e endossados quando comparados a itens redigidos usando palavras com valência afetiva neutra ou ambígua (Bäckström, Björklund, \& Larsson, 2012). Por exemplo, um item que afirma "Eu sou uma pessoa comunicativa" pode eliciar mais RSD do que um que afirma algo semelhante, mas de forma menos explicitamente valorativa, como seu correspondente "Converso até com pessoas estranhas", que é ambíguo quanto ao valor social. Portanto, pode haver vantagens na redação cautelosa de itens evitando um tom pejorativo ou excessivamente positivo. Esse cuidado proporciona um método de prevenção de RSD, a neutralização valorativa, solução adotada no presente estudo no que diz respeito a dois instrumentos de traços de personalidade.

A neutralização valorativa é uma forma de controlar a desejabilidade social por meio da reescrita dos itens. 0 método foi desenvolvido por Bäckström (2007) e colaboradores (Bäckström \& Björklund, 2013, 2014, 2016; Bäckström, Björklund, \& Larsson, 2009, 2014), com base nos estudos de Peabody (1967), Jackson (1984) e Paulhus (1988), que destacam a importância do cuidado com as palavras selecionadas para a construção de um item e a representação social delas. A premissa da neutralização valorativa é a possibilidade de passar a mesma informação usando palavras menos sujeitas à desejabilidade social - dito de maneira coloquial, palavras mais "amenas". Essa premissa é aplicada à construção de itens, o que significa que o foco é a prevenção das RSD a partir da escrita cuidadosa dos itens, em vez de sua remediação 


\section{H. INTERACÃO EM 2. PSICOLOGIA}

a posteriori. Esse tipo de abordagem preventiva foi denominado, por Paulhus (1988), como método de "controle racional" de RSD.

A neutralização valorativa é implementada por meio de diversas etapas. Em primeiro lugar, deve-se partir de um bom conjunto de itens, ou seja, que sejam adequados indicadores do atributo latente de interesse. Nesse caso, é possível tanto escrever itens, e contar com a avaliação semântica por parte de juízes especialistas na área que avaliem a pertinência dos itens, quanto usar um instrumento já existente e com evidências de validade. Após, deve-se coletar informação sobre a desejabilidade social do conteúdo dos itens. Tipicamente, uma amostra de pessoas da população geral é convidada a avaliar o quão desejável é manifestar a característica ou o comportamento descrito. Para esse fim pode ser usada uma escala Likert em que o enunciado de cada item é classificado como 1 = Totalmente indesejável e 9 = Totalmente desejável . Na sequência, são calculadas médias dessas avaliações para cada item, informação que representa a magnitude do seu conteúdo valorativo. Por exemplo, um item com média 1,5 é um item que foi avaliado como extremamente indesejável, enquanto outro com média 8,6 representa um atributo altamente desejável. Já um item com média próxima a 5 não é desejável e nem indesejável, sendo tipicamente avaliado como neutro ou ambíguo em termos de valor social.

A etapa subsequente é a mais importante do processo. Ela consiste em reescrever os itens com médias próximas aos extremos 1 ou 9, que são aqueles mais explicitamente indesejáveis ou desejáveis, respectivamente. 0 intuito é identificar, no enunciado do item, as palavras com valência fortemente negativa ou positiva, tentando substitui-las por outras mais neutras ou menos impactantes (Bäckström et al., 2012). O ponto chave é diminuir o conteúdo valorativo, mas manter o conteúdo descritivo, sem fazer com que o item deixe de avaliar o fator de interesse. Por exemplo, o item sobre o fator Conscienciosidade "Sou uma pessoa que tende a ser desorganizada" contém a palavra desorganizada, que tem um valor social indesejável. Esse item pode ser rescrito como "Às vezes, deixo as coisas fora do lugar", que emprega palavras menos pejorativas para expressar um conteúdo descritivo similar. Apesar de sutil a diferença, ela é capaz de reduzir as RSD por parte dos indivíduos examinandos (Bäckström \& Björklund, 2013). Assim, para certificar que houve alteração no julgamento da desejabilidade do item após reescrevê-lo, é necessário submetê-lo a uma nova rodada de avaliação por pessoas da população geral (juízes). Esse processo deve ser reiterado até que o conteúdo dos itens seja reduzido o máximo possível. No presente estudo, adotou-se o critério de que a média geral dos itens deve ser próxima a $5 \mathrm{com}$ um desviopadrão menor do que 1 para serem considerados neutralizados, como descrito mais adiante.
Ariela Raissa Lima Costa e Nelson Hauck Filho

Uma preocupação legítima quanto ao procedimento da neutralização valorativa diz respeito à manutenção das características psicométricas dos itens neutralizados. Dado que minimizar o conteúdo valorativo significa remover variância compartilhada entre os itens, um revés que poderia ser hipotetizado é que o procedimento impacta na fidedignidade e nas associações do instrumento com variáveis externas. Não obstante, Bäckström e Björklund (2013) mostraram que a neutralização valorativa de um inventário dos Cinco Grandes Fatores (IPIP-100) resultou em um instrumento com fidedignidade aceitável e mesma estrutura fatorial que sua versão original não neutralizada. Em outro estudo, Bäckström, Björklund e Larsson (2014), encontraram que uma versão neutralizada do IPIP-300 (um inventário baseado no NEO-PI-R) apresentou correlações com uma série de variáveis externas muito similares àquelas apresentadas pela versão original do instrumento. Em resumo, as evidências sugerem que remover a variância valorativa de um instrumento não impacta nas propriedades psicométricas se for mantida a variância descritiva, relacionada ao atributo de interesse. Portanto, a neutralização valorativa é um procedimento viável, vantajoso e psicometricamente justificável de reduzir RSD na avaliação via autorrelato de traços de personalidade.

A redução das RSD a um instrumento psicométrico é um benefício com diversas potencialidades em contextos de pesquisas e aplicação cotidiana, uma vez que pode refinar a avaliação de traços da personalidade. Todavia, esse procedimento ainda é pouco utilizado na literatura, com nenhuma aplicação, até onde se pode apurar, no contexto brasileiro. Em virtude disso, baseando-se na proposta de Bäckström (2007), este trabalho teve como objetivo elaborar uma versão menos socialmente desejável dos instrumentos Big Five Inventory (BFI), que avalia os Cinco Grandes Fatores da personalidade, e a IPIP-Modesty Scale (IPIP-MS), uma escala de modéstia. Tendo em vista que o procedimento adotado tem como alvo remover a variância valorativa, foram hipóteses que: 1) haveria correlação alta e positiva entre as versões original e neutralizada de cada instrumento; 2) os instrumentos neutralizados se correlacionariam menos com um instrumento avaliativo de desejabilidade social em comparação às suas versões originais; e 3) seria mantida a estrutura fatorial de ambos os instrumentos após a neutralização dos itens.

\section{MÉTODO}

\section{PARTICIPANTES}

Primários (avaliação da desejabilidade dos itens). A amostra foi de 50 participantes da população geral, com média de idades de 29,16 anos $(D P=8,27)$, sendo $54,24 \% \mathrm{mu}$ - 


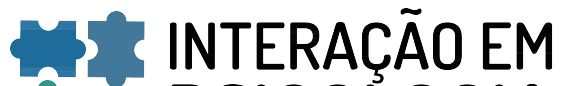 ET PSICOLOGIA}

Iheres. Esses participantes serviram como juízes que classificaram os itens quanto à desejabilidade social do conteúdo. A inclusão desses participantes se fundamenta na necessidade de garantir que a reescrita dos itens de fato reduza o componente valorativo.

Secundários (análise dos itens neutralizados). A amostra foi de 379 universitários, entre 18 e 47 anos $(M=22,53$ anos; $D P=6,2$ ), sendo $58 \%$ homens. Os participantes eram $73,1 \%$ brancos, $20,1 \%$ pardos e $5,5 \%$ negros. Em relação à renda $51 \%$ declararam possuir de 1 a 5 salários mínimos, $27,5 \%$ de 6 a 10 salários mínimos, $7,5 \%$ de 11 a 15 salários mínimos, e $6,3 \%$ mais de 15 salários mínimos. Em relação ao relacionamento, $49,7 \%$ eram solteiros, 35,4\% namoravam, e 13\% eram casados. Em relação à escolaridade dos pais, 30,3\% e 29,3\% declararam que seus pais e mães tinham ensino médio completo, respectivamente.

\section{INSTRUMENTOS}

Big Five Inventory (BFI - Benet-Martinez \& John, 1998). O BFI é um inventário que avalia os cinco grandes fatores da personalidade, sendo composto por cinco subescalas referente aos fatores Concienciosidade $(a=0,78)$, Socialização ( $a$ $=0,70)$, Neuroticismo $(a=0,79)$, Extroversão $(a=0,77)$ e Abertura $(a=0,76)$. A escala de resposta dos itens é do tipo Likert, $1=$ Discordo totalmente a $5=$ Concordo totalmente. $\mathrm{A}$ tradução brasileira do BFI foi realizada por Santos e Primi (2014).

Escala de Modéstia do International Personality of Item Pool (IPIP-EM-A5, do NEO-PI; Goldberg et al., 2006). A escala é uma representação da faceta modéstia (A5) baseado no NEO-PI, extraída do International Personality Item Pool (IPIP). $O$ instrumento contém 10 itens que avaliam traços de modéstia $(a=0,77)$. A escala de resposta dos itens é do tipo Likert, 1 = Discordo totalmente e 5 = Concordo totalmente. A tradução dos itens foi realizada pelos autores do presente estudo, sendo verificada a adequação dos termos para a cultura brasileira, e seguida de tradução reversa por um terceiro pesquisador alheio ao objetivo do instrumento, conforme recomendações da literatura (Borsa, Damásio, \& Bandeira, 2012).

Escala de Manejo de impressão e Autoengano (Impression Management/Self-Deception Scales - IPIP-IMSD, PAS: PauIhus, 1991, Goldberg et al., 2006). A escala é uma representação da escala Inventário Balanceado de Respostas Desejáveis (IBRD) de Paulhus (1991), extraída do International Personality Item Pool (IPIP). Trata-se de um instrumento de autorrelato de 30 itens, que avaliam duas dimensões da desejabilidade social: (a) Manejo de impressão, uma tendência de produzir distorções conscientes nas respostas, com o propósito de causar uma boa impressão ( $a=0.82$ ); e (b) Autoengano, uma distorção na percepção das próprias características $(a=0.80)$. A escala de resposta dos itens é do tipo Likert, $1=$
Ariela Raissa Lima Costa e Nelson Hauck Filho

Discordo totalmente a 5 = Concordo totalmente. A tradução da IPIP-IMSD foi realizada pelo grupo de pesquisa do segundo autor deste trabalho, seguindo os mesmos cuidados tidos para com a escala de modéstia descrita anteriormente.

\section{PROCEDIMENTOS}

O estudo foi conduzido em duas etapas. Na primeira etapa, os itens de todos os instrumentos foram submetidos à avaliação de desejabilidade social por parte dos participantes primários do estudo. Foi utilizada uma escala de resposta do tipo Likert de 1 = Totalmente indesejável a $9=$ Totalmente desejável. 0 objetivo desse procedimento foi identificar quais itens apresentavam conteúdo valorativo mais extremado, sendo caracterizados como tal aqueles com médias próximas a 1 ou 9 . Na sequência, esses itens identificados foram reescritos segundo a proposta de neutralização valorativa (Bäckström, 2007), e submetidos, novamente, aos participantes primários para verificar se houve alguma alteração na desejabilidade social dos enunciados. Isso foi repetido oito vezes, até que todos os itens tivessem sido neutralizados, conforme alguns critérios descritos mais adiante. Vale ressaltar que alguns itens foram neutralizados já na primeira rodada, enquanto outros necessitaram de modificações sucessivas. Itens já neutralizados não foram mais apresentados aos participantes primários nas rodadas subsequentes.

$\mathrm{Na}$ segunda etapa, os itens originais e neutralizados foram aplicados aos participantes secundários. Os participantes secundários, diferentemente dos juízes, responderam aos itens sob a instrução usual de usar a escala Likert padrão do instrumento para efetuar uma autoavaliação. Ou seja, os participantes secundários usaram as escalas para fornecer um autorrelato de sua própria personalidade, como usual em pesquisas na área, indicando o quanto concordavam ou discordavam do enunciado de cada item ( 1 = Discordo totalmente a 5 = Concordo totalmente).

Foram empregadas estratégias mistas de coleta de dados. Os participantes primários foram convidados pela internet via e-mail e rede social (Facebook), em que Ihes foi enviado um link do site Survey Monkey. No início do questionário online, foi fornecida uma breve explicação sobre o objetivo da pesquisa, além de um exemplo de análise esperada dos participantes visando a uma solidificação da explicação anterior. Em seguida, o instrumento foi apresentado, sendo o participante convidado a classificar a desejabilidade social de uma série de itens de autorrelato. Ao final, foram solicitadas apenas informações demográficas de sexo e idade. Os participantes secundários, por sua vez, foram avaliados de forma presencial e coletiva, em sala de aula, em uma universidade particular do interior de São Paulo. A pesquisa foi aprovada pelo Comitê de Ética da Universidade São Francisco, CAAE n. 53659515.2.0000.5514. 


\section{W INERACÄOEM ET PSICOLOGIA}

\section{PLANO DE ANÁLISE DE DADOS}

Para a primeira etapa, foram realizadas análises descritivas de média e desvio padrão, com o software R, pacote psych (Revelle, 2014). Como já descrito anteriormente, assumiuse como critério principal de neutralização que cada item apresentasse média o mais próximo possível de 5 após as rodadas de neutralização e classificação por parte dos participantes primários. 0 procedimento foi encerrado após o conjunto de itens obter uma média geral próxima a 5 , e um desvio-padrão menor do que 1 , indicando a presença de poucos itens mais distantes do ponto central da escala de 9 pontos usada para classificar os itens.

Outra perspectiva adotada para avaliar a qualidade do procedimento de neutralização foi uma regressão linear simples. Testou-se, em cada rodada, se o polo descritivo dos itens (variável binária, sendo cada item codificado como +1 se seu conteúdo descritivo era positivamente relacionado ao traço, e -1 se era negativamente relacionado) estava relacionado à desejabilidade social desses itens (variável critério), ou seja, as médias obtidas para os itens mediante a avaliação feita pelos participantes primários. Em linguagem $\mathrm{R}$, a regressão foi, simplesmente, "polo_descritivo = notas_desejabilidade", ou seja, dois vetores em que cada valor representa um item distinto. A expectativa era que o polo descritivo de cada item não estivesse relacionado às notas de desejabilidade, ou seja, que itens do polo inferior do traço não fossem, consistentemente, avaliados como indesejáveis (ou desejáveis) e viceversa. Sendo isso obtido, a análise de regressão com as duas variáveis deveria revelar um beta $(\beta)$ e um $R^{2}$ próximos a 0 , pois eles indicam o quanto o polo descritivo dos itens é explicado pela desejabilidade social. Assim, quanto menor o valor, menor a influência da desejabilidade social.

Para a segunda etapa, foram analisadas estatísticas descritivas dos itens (média e desvio padrão) e a consistência interna dos instrumentos por meio do coeficiente alfa. As versões neutralizadas dos instrumentos BFI e IPIP-EM-A5 foram correlacionadas às suas respectivas versões originais, sendo esperadas correlações positivas e de magnitude, pelo menos, moderada. Foram também comparadas as associações entre cada versão dos instrumentos (original versus neutralizada) com o instrumento IPIP-IMSD. A significância para a diferença entre os coeficientes de correlação observados foi testada pelo procedimento $r$-para-z de Fisher, implementado a partir da calculadora online disponível em: vassarstats.net/rdidd.html. Posteriormente, foi rodada uma análise fatorial exploratória para investigar a estrutura fatorial das versões original e neutralizada do BFI e da IPIP-EM-A5. Foi empregado o estimador quadrados mínimos ponderados robustos (Weighted Least Squares Mean- and Variance-adjusted), em combinação a uma matriz de correlações policóri-
Ariela Raissa Lima Costa e Nelson Hauck Filho

cas. A rotação foi oblíqua Geomin, pois mesmo controlando o aspecto valorativo, os fatores ainda poderiam apresentar algum grau de relacionamento. Foi empregado o programa MPlus 7.11.

\section{RESULTADOS}

A primeira parte das análises contemplou a etapa de neutralização dos itens. Ao todo, foram oito rodadas até obter resultados de acordo com os critérios descritos anteriormente, o que pode ser visto na Tabela 1. Como esperado, ao longo das rodadas, houve uma diminuição gradativa na saliência do conteúdo valorativo, informação que se confirma a partir das médias de desejabilidade dos itens, dos desvios padrão, dos coeficientes de regressão e dos coeficientes de determinação. Os instrumentos originais, apesar de apresentarem uma média geral próxima a $5(M=5,74)$, possuíam muitos itens distantes dessa média $(D P=2,32)$, além de uma clara conexão entre polo descritivo de seus itens e a desejabilidade social. Isto é, altos escores nas escalas originais (exceto neuroticismo, ao contrário) coincidem com características desejáveis, e baixos escores coincidem com aspectos negativos, e vice-versa; $\beta=0,54, p<0,001, R^{2}=0,29$. As versões finais neutralizadas dos instrumentos apresentaram média 4,87 e desvio padrão 0,55 , havendo uma quase ortogonalidade entre conteúdo descritivo e valorativo nesses itens, $\beta=$ $0,13, R^{2}=0,05$. As medidas de cada rodada poder ser observada na Tabela 1. A diminuição dessas medidas sugere um favorecimento do aspecto descritivo em detrimento do aspecto valorativo na interpretação do conteúdo dos itens.

$\mathrm{Na}$ segunda etapa, foram aplicados, a uma amostra de universitários, os itens originais e aqueles neutralizados, com o objetivo de verificar as qualidades descritivas e psicométricas dos novos instrumentos.

\section{Tabela 1. Medidas descritivas dos itens neutralizados} por rodada

\begin{tabular}{lccccc}
\hline Rodadas de neutralização & $k$ & $M$ & $D P$ & $\beta$ & $R^{2}$ \\
\hline Escalas originais & 54 & 5,74 & 2,32 & $0,54^{\star \star \star}$ & 0,29 \\
1 & 54 & 5,87 & 1,37 & $0,18^{\star \star}$ & 0,03 \\
2 & 54 & 5,21 & 0,85 & $-0,01 * \star$ & 0,00 \\
3 & 54 & 5,18 & 0,68 & $0,03^{\star \star}$ & 0,00 \\
4 & 54 & 5,17 & 0,64 & $0,03^{\star \star}$ & 0,00 \\
5 & 54 & 5,13 & 0,63 & $0,12^{\star \star}$ & 0,01 \\
6 & 54 & 4,72 & 0,7 & $0,39 *$ & 0,15 \\
7 & 54 & 4,89 & 0,63 & $0,22^{\star}$ & 0,12 \\
8 & 54 & 4,87 & 0,55 & $0,13^{\star \star}$ & 0,05 \\
\hline Nota: $k=$ número de itens, $M=$ média, $D P=$ desvio padrão, \\
$\beta=$ coeficiente de regressão, $\mathrm{R}^{2}=$ coeficiente de determinação. \\
${ }^{\star} p<0,01$.
\end{tabular}




\section{- INTERACÃO EM PSTICOLOGIA}

Os dados coletados com essa segunda amostra foram analisados, e são relatados a seguir.

Na Tabela 2, são apresentados os valores da consistência interna por fator dos instrumentos originais e neutralizados.
Devido à redução de variância comum via neutralização valorativa, a consistência interna das escalas sofreu um ligeiro decréscimo em relação aos itens originais.

$\mathrm{Na}$ sequência, foram analisadas as correlações entre os

Tabela 2. Consistência interna, média e desvio padrão dos instrumentos originais e neutralizados

\begin{tabular}{|c|c|c|c|c|c|c|}
\hline \multirow[b]{2}{*}{ Fatores } & \multicolumn{3}{|c|}{ Originais } & \multicolumn{3}{|c|}{ Neutralizados } \\
\hline & $a$ & $\mathrm{M}$ & SD & $a$ & $\mathrm{M}$ & SD \\
\hline Extroversão & 0,78 & 3,3 & 0,72 & 0,65 & 2,8 & 0,68 \\
\hline Conscienciosidade & 0,69 & 3,5 & 0,58 & 0,41 & 3,0 & 0,5 \\
\hline Socialização & 0,59 & 3,7 & 0,54 & 0,43 & 3,1 & 0,53 \\
\hline Neurotiscismo & 0,83 & 3,0 & 0,84 & 0,64 & 3,4 & 0,65 \\
\hline Abertura & 0,70 & 3,5 & 0,61 & 0,59 & 3,1 & 0,59 \\
\hline Modéstia & 0,77 & 2,6 & 0,69 & 0,74 & 2,9 & 0,69 \\
\hline
\end{tabular}

fatores dos instrumentos originais e neutralizados, buscando avaliar se, de fato, a neutralização produziu instrumentos altamente correlacionados às suas versões originais. Na primeira e na segunda parte da Tabela 3, respectivamente, estão apresentadas as correlações dos fatores entre si nas versões original e neutralizada dos instrumentos. Percebe-se que a magnitude das correlações entre fator foi atenuada na versão neutralizada do $\mathrm{BFI}$, o que se explica pela redução na variância valorativa. Ainda que as correlações já não fossem altas entre os fatores do BFI original, os coeficientes da versão neutralizada estiveram anda mais próximos de zero. $\mathrm{Na}$ terceira parte da Tabela 3, estão os coeficientes de validade

Tabela 3. Correlação obtida para os instrumentos BFI e IPIP nas versões originale neutralizada

\begin{tabular}{|c|c|c|c|c|c|c|}
\hline \multirow[b]{2}{*}{ Itens originais } & \multicolumn{6}{|c|}{ Itens originais } \\
\hline & $\mathrm{E}$ & C & $\mathrm{S}$ & $\mathrm{N}$ & $A$ & $\mathrm{M}$ \\
\hline Conscienciosidade & 0,13 & - & & & & \\
\hline Socialização & 0,08 & 0,2 & - & & & \\
\hline Neuroticismo & $-0,18$ & $-0,16$ & $-0,32$ & - & & \\
\hline Abertura & 0,29 & 0,16 & 0,18 & $-0,09$ & - & \\
\hline \multirow[t]{2}{*}{ Modéstia } & $-0,35$ & 0,03 & 0,15 & 0,11 & $-0,15$ & - \\
\hline & \multicolumn{6}{|c|}{ Itens neutralizados } \\
\hline Itens neutralizados & En & $\mathrm{Cn}$ & Sn & $\mathrm{Nn}$ & An & $\mathrm{Mn}$ \\
\hline Conscienciosidade & 0,01 & - & & & & \\
\hline Socialização & $-0,06$ & 0,04 & - & & & \\
\hline Neuroticismo & 0,09 & 0,06 & $-0,10$ & - & & \\
\hline Abertura & 0,16 & $-0,08$ & 0,18 & 0,04 & - & \\
\hline \multirow[t]{2}{*}{ Modéstia } & $-0,35$ & 0,04 & 0,09 & 0,05 & $-0,18$ & - \\
\hline & \multicolumn{6}{|c|}{ Itens neutralizados } \\
\hline Itens originais & En & $\mathrm{Cn}$ & $\mathrm{Sn}$ & $\mathrm{Nn}$ & An & $\mathrm{Mn}$ \\
\hline Extroversão & $0,77^{*}$ & $-0,01$ & $-0,12$ & 0,07 & 0,13 & $-0,31$ \\
\hline Conscienciosidade & $-0,03$ & $0,55^{\star}$ & 0,02 & 0,01 & $-0,1$ & $-0,03$ \\
\hline Socialização & 0,05 & 0,18 & $0,49 *$ & $-0,09$ & 0,02 & 0,12 \\
\hline Neuroticismo & $-0,11$ & $-0,02$ & $-0,08$ & $0,68 *$ & 0,07 & 0,03 \\
\hline Abertura & 0,19 & 0,04 & 0,12 & $-0,03$ & $0,59 *$ & $-0,22$ \\
\hline Modéstia & $-0,33$ & $-0,01$ & 0,20 & 0,11 & $-0,08$ & $0,69 *$ \\
\hline
\end{tabular}




\section{H NTERAC̄OOEM ET PSICOLOGIA}

convergente. Como hipotetizado, as correlações entre os fatores das versões originais e neutralizadas foram todas positivas, de magnitude moderada (0,49 para socialização) a alta $(0,77$ para extroversão). Ou seja, a neutralização reduziu a variância valorativa, mas manteve a variância descritiva contida nos itens, sustentando a proposta de que os itens neutralizados continuaram avaliando os mesmos fatores dos instrumentos originais.

Ainda outra hipótese correlacional foi testada. Considerando que o aspecto valorativo foi controlado nas versões

Tabela 4. Correlação entre os instrumentos e desejabilidade social

\begin{tabular}{lcccccc}
\hline & \multicolumn{3}{c}{ Originais } & \multicolumn{3}{c}{ Neutralizados } \\
\hline Fatores & $\mathrm{MI}$ & $\mathrm{AE}$ & Total & $\mathrm{MI}$ & $\mathrm{AE}$ & Total \\
\hline Extroversão & $-0,05$ & 0,35 & 0,13 & $-0,09$ & $0,20^{\mathrm{a}}$ & 0,02 \\
Conscienciosidade & 0,43 & 0,32 & 0,50 & $0,26^{\mathrm{b}}$ & $0,14^{\mathrm{a}}$ & $0,02^{\mathrm{b}}$ \\
Socialização & 0,41 & 0,11 & 0,39 & $0,26^{\mathrm{a}}$ & $-0,16^{\mathrm{b}}$ & $-0,30^{\mathrm{b}}$ \\
Neuroticismo & $-0,18$ & $-0,46$ & $-0,36$ & $0,04^{\mathrm{b}}$ & $-0,30^{\mathrm{b}}$ & $-0,11^{\mathrm{b}}$ \\
Abertura & 0,09 & 0,17 & 0,15 & $-0,06^{\mathrm{a}}$ & $-0,01^{\mathrm{b}}$ & $-0,06^{\mathrm{a}}$ \\
Modéstia & 0,33 & $-0,44$ & 0,06 & $0,21^{\mathrm{a}}$ & $-0,34^{\mathrm{a}}$ & $0,01^{\mathrm{b}}$ \\
\hline Nota. Ml=manejo de impressão, AE= autoengano. & & & \\
a diferença entre coeficientes significativa no nível de $p<0,01$. & & &
\end{tabular}

para quase todos os coeficientes de correlação (exceto para dos envolvendo o fator extroversão do BFI). Em alguns casos, o sinal da correlação até mudou de sentido, embora tenha se mantido próximo a zero. Isso ocorreu para o fator neuroticismo, e apenas indica que a versão neutralizada resultou ligeiramente mais desejável do que a versão original, que continha itens extremamente indesejáveis socialmente. Isso confirma o sucesso da neutralização valorativa dos itens, uma vez que possibilita uma redução nos seus componentes valorativos, responsáveis por eliciar as RSD.

Afinal, investigou-se se a estrutura fatorial dos instrumentos BFI e Escala de Modéstia permaneceria a mesma nas ver- neutralizadas, espera-se que elas se correlacionem menos com instrumentos que avaliam desejabilidade social. Na Tabela 4, é possível comparar as correlações dos instrumentos originais e neutralizados com uma escala de desejabilidade social. Mais especificamente, a tabela mostra a associação das duas versões de cada instrumento BFI e IPIP-EM-A5 com a Escala de Manejo de Impressão e Autoengano de Paulhus. Para verificar se a diminuição da relação das versões neutralizadas com desejabilidade social não se deveu ao acaso, foi calculada a significância estatística da diferença entre as correlações. Houve uma redução estatisticamente significativa sões neutralizadas. Para o BFI e sua versão neutralizada, foram empregadas duas estratégias de análise: com todos os itens e com uma seleção menor de indicadores. Mais detaIhadamente, o BFI original foi avaliado por meio de uma análise fatorial exploratória com todos os 44 itens, e outra com 15 parcelas formadas pela soma de trio de itens (14 trios e uma dupla). Por sua vez, uma análise exploratória buscou encontrar a solução composta pelos melhores itens neutralizados do BFI. Para a IPIP-EM-A5, foi conduzida apenas uma análise com todos os itens. Os resultados podem ser encontrados na Tabela 5. Como visto, tanto para o BFI quanto para a IPIP-EMA5, o ajuste aos dados foi bastante modesto quando consi-

Tabela 5. Análise fatorial exploratória dos instrumentos originais e neutralizados

\begin{tabular}{lccccc}
\hline Instrumento/versão & $x^{2}$ & $g l$ & RMSEA & CFI & TLI \\
\hline BFI (cinco fatores oblíquos) & & & & & \\
\hline 44 itens originais & $1389,19^{*}$ & 736 & 0,048 & 0,890 & 0,858 \\
15 parcelas de itens originais & $71,70^{\star}$ & 40 & 0,046 & 0,976 & 0,928 \\
44 itens neutralizados & $1352,06^{\star}$ & 736 & 0,047 & 0,833 & 0,786 \\
20 melhores itens neutralizados & $190,65^{\star}$ & 100 & 0,049 & 0,958 & 0,921 \\
\hline IPIP - Escala de Modéstia (um fator) & & & & & \\
\hline 10 itens originais & $205,31^{\star}$ & 26 & 0,135 & 0,901 & 0,829 \\
10 itens neutralizados & $344,52^{\star}$ & 26 & 0,181 & 0,883 & 0,797 \\
\hline
\end{tabular}

Nota. $\mathrm{gl}=$ graus de liberdade, RMSEA = Root Mean Square Error of Approximation, $\mathrm{CFI}=$ Confirmatory Fit Index, TLI = Tucker-Lewis Index, BFI = Big Five Inventory.

${ }^{\star} p<0,05$ 


\section{H INERACÃOEM ET. PSICOLOGIA}

derados todos os itens originais aplicados aos modelos propostos na literatura (cinco fatores oblíquos para o BFI, e um fator apenas para a IPIP-EM-A5). Os índices de modificação foram sugestivos de que a falta de ajuste se deveu, principalmente, à presença de correlações residuais entre itens com escrita similar, fenômeno conhecido como "dependência local". Confirmando essa hipótese, um modelo de cinco fatores oblíquos do BFI utilizando com 15 parcelas em vez dos 44 itens originais apresentou um bom ajuste aproximado aos dados.

No que diz respeito à análise dos instrumentos neutralizados, os resultados foram promissores. Em primeiro lugar, am- bas as versões neutralizadas dos instrumentos BFI e IPIP-MSA5, quando analisadas com todos os seus itens, apresentaram um ajuste aos dados similar àquele encontrado para os instrumentos originais. No caso do BFI neutralizado, foi ainda possível fazer uma seleção com os melhores 20 itens, a fim de compor um instrumento breve. Um modelo de cinco fatores oblíquos para esse conjunto seleto de itens apresentou um bom ajuste aproximado aos dados, de maneira similar ao encontrado para o $\mathrm{BFI}$ ao empregar 15 parcelas de itens. A Tabela 6 apresenta as cargas fatoriais para esses 20 itens neutralizados nos cinco fatores oblíquos extraídos. Para fins de melhor entendimento, foram incluídos na análise

Tabela 6. Análise fatorial exploratória dos 20 melhores itens neutralizados do BFI

\begin{tabular}{|c|c|c|c|c|c|}
\hline & $\mathrm{F} 1$ & F2 & F3 & $\mathrm{F} 4$ & F5 \\
\hline $\begin{array}{l}\text { Raramente me canso fazendo várias } \\
\text { atividades }\end{array}$ & $-0,408$ & 0,131 & 0,231 & 0,195 & 0,009 \\
\hline Empolgo-me com quase tudo. & 0,783 & 0,022 & 0,027 & $-0,183$ & $-0,137$ \\
\hline $\begin{array}{l}\text { Evito revidar mesmo quando as pessoas me } \\
\text { ofendem. }\end{array}$ & 0,879 & 0,051 & 0,031 & $-0,082$ & $-0,083$ \\
\hline $\begin{array}{l}\text { Acontece de eu falar as coisas de um jeito } \\
\text { indelicado. }\end{array}$ & $-0,308$ & 0,067 & 0,126 & 0,005 & $-0,351$ \\
\hline Às vezes deixo as coisas fora do lugar. & 0,056 & 0,088 & 0,202 & 0,625 & $-0,028$ \\
\hline $\begin{array}{l}\text { Teimo em fazer coisas até o fim, mesmo } \\
\text { quando isso me desgasta. }\end{array}$ & $-0,089$ & $-0,050$ & 0,216 & 0,596 & 0,066 \\
\hline Tenho uma frieza frente ao perigo. & 0,142 & $-0,102$ & $-0,021$ & 0,433 & $-0,084$ \\
\hline $\begin{array}{l}\text { Sei mais sobre arte e música do que as outras } \\
\text { pessoas. }\end{array}$ & 0,096 & $-0,037$ & 0,292 & $-0,434$ & 0,021 \\
\hline Preocupo-me muito. & 0,509 & 0,651 & $-0,004$ & 0,052 & 0,174 \\
\hline $\begin{array}{l}\text { Sou uma pessoa de extrema tranquilidade, } \\
\text { mesmo quando não deveria. }\end{array}$ & 0,003 & $-0,575$ & 0,642 & 0,009 & 0,083 \\
\hline Sou uma pessoa emotiva. & 0,067 & $-0,646$ & 0,580 & $-0,009$ & 0,102 \\
\hline Rotina é muito importante para mim. & 0,469 & 0,648 & 0,078 & 0,145 & 0,092 \\
\hline Confio demais nas pessoas. & $-0,152$ & 0,071 & 0,060 & $-0,013$ & $-0,474$ \\
\hline Sei me desligar dos problemas dos outros. & $-0,063$ & 0,218 & $-0,008$ & 0,102 & 0,329 \\
\hline Sigo meus planos de maneira inflexível. & $-0,050$ & 0,392 & 0,001 & $-0,075$ & $-0,233$ \\
\hline $\begin{array}{l}\text { Diversas coisas atraem minha atenção ao } \\
\text { mesmo tempo. }\end{array}$ & 0,260 & 0,182 & 0,204 & $-0,023$ & 0,441 \\
\hline Sempre invento histórias na minha cabeça. & $-0,005$ & 0,004 & 0,562 & 0,013 & $-0,078$ \\
\hline $\begin{array}{l}\text { Gosto de criar coisas novas, mesmo que não } \\
\text { tenham utilidade. }\end{array}$ & $-0,061$ & $-0,244$ & 0,474 & 0,110 & $-0,041$ \\
\hline Sou acessível para qualquer pessoa. & 0,018 & 0,425 & 0,376 & $-0,017$ & $-0,131$ \\
\hline Possuo poucos interesses artísticos. & 0,002 & 0,095 & 0,355 & $-0,104$ & 0,058 \\
\hline Extroversão & $-0,846$ & 0,166 & 0,135 & $-0,039$ & $-0,006$ \\
\hline Socialização & $-0,078$ & 0,101 & 0,054 & $-0,235$ & 0,652 \\
\hline Conscienciosidade & $-0,009$ & $-0,033$ & 0,112 & $-0,802$ & $-0,005$ \\
\hline Neuroticismo & $-0,007$ & $-0,786$ & 0,284 & 0,176 & $-0,070$ \\
\hline Abertura & $-0,170$ & 0,195 & 0,488 & $-0,143$ & $-0,026$ \\
\hline
\end{tabular}

Nota. Cargas fatoriais acima de 0,30 foram colocadas em negrito para facilitar a interpretação da solução fatorial. 


\section{U NTERAC̄öEM PSICOLOGIA}

para essa tabela os escores brutos dos cinco grandes fatores, calculados a partir dos itens originais do BFI. Apesar da presença de algumas cargas fatoriais cruzadas, essa inclusão confirma a interpretação de que os fatores extraídos são equivalentes aos cinco grandes fatores da personalidade. 0 ajuste para essa análise suplementar foi razoável, $x^{2}(185)=$ $376,08, \mathrm{RMSEA}=0,052, \mathrm{CFI}=0,931, \mathrm{TLI}=0,887$.

A Tabela 7, por sua vez, inclui as cargas fatoriais estima-

Tabela 7. Análise fatorial dos itens neutralizados da Escala de Modéstia

\begin{tabular}{lc}
\hline & F1 \\
\hline Desagrada-me ser o assunto principal da conversa. & $-0,318$ \\
Desagrada-me falar sobre mim mesmo. & $-0,408$ \\
Considero-me uma pessoa comum. & $-0,306$ \\
Acho pouco importante fazer os outros notarem minhas qualidades. & $-0,332$ \\
Costumo ter mais habilidades do que os outros. & 0,644 \\
Sou uma pessoa mais interessante do que a maioria. & 0,697 \\
Tendo a enxergar mais minhas qualidades do que meus defeitos. & 0,396 \\
Gosto de mostrar o quanto sei sobre algo. & 0,830 \\
Gosto que saibam sobre minhas capacidades. & 0,885 \\
Gosto quando falam a meu respeito em uma conversa. & 0,554 \\
\hline
\end{tabular}

\section{DISCUSSÃO}

A presente pesquisa teve como objetivo aplicar a técnica da neutralização valorativa nos itens de dois instrumentos que avaliam personalidade, BFI e IPIP-EM-A5. Apesar de terem sido necessárias diversas rodadas de neutralização, foi possível obter um conjunto neutralizado de itens que preservou, dentro do possível, o conteúdo descritivo dos itens originais. De maneira geral, os resultados sustentaram as hipóteses levantadas, mostrando que os instrumentos neutralizados: 1) se correlacionaram positivamente, em magnitude de moderada a forte, com suas respectivas versões originais, 2) se correlacionaram significativamente menos com um instrumento avaliativo de desejabilidade social, e 3) apresentaram a mesma estrutura fatorial que seus respectivos instrumentos originais.

Em conformidade com as expectativas, as versões originais e neutralizadas dos instrumentos se correlacionaram em grau moderado a alto. Apesar de positivas e significativas, as correlações não foram perfeitas porque o procedimento de neutralização em si representa uma diminuição da variância irrelevante, aqui representada pelo conteúdo valorativo, nos escores da versão neutralizada. A variância irrelevante é composta por tudo que influencia a resposta ao item, mas que não tem relação com o traço latente avaliado (American Educational Research Association, American Psychological Association \& National Council on Measurement in Education, das para os itens neutralizados da Escala de Modéstia no modelo de um fator testado. Como visto, todos os itens apresentaram carga acima de 0,30, sendo bastante altas em alguns casos (e.g., item NHU_09, com carga 0,89). Mais uma vez, os resultados evidenciam a viabilidade da neutralização proposta. Vale observar que as cargas fatoriais mais modestas ficaram por conta dos itens sobre falta de modéstia (false keyed ou invertidos).
2014; Messick, 1995). Confirmando essa interpretação, os menores coeficientes de validade convergente ocorreram exatamente para os fatores mais sujeitos à desejabilidade social no modelo dos Cinco Grandes Fatores, ou seja, a conscienciosidade e a socialização $(0,49$ e 0,55). Essas dimensões dizem respeito a características altamente valorizadas socialmente, tais como asseio, dedicação, planejamento, generosidade, bondade e boas maneiras. Naturalmente, esses são fatores para os quais se espera uma maior parcela de variância valorativa ou irrelevante.

Outro resultado favorável é que as correlações com as dimensões da desejabilidade social foram menores para os instrumentos neutralizados relativamente aos originais. Esse padrão foi observado nos estudos de Bäckström e Björklund $(2013,2014)$. À exceção do fator extroversão do BFI, os resultados confirmaram amplamente as expectativas, sugerindo que os itens neutralizados tendem a produzir menores associações com inventários avaliativos das dimensões da desejabilidade social. Novamente, a interpretação é que o ocorrido se deve à diminuição da variância irrelevante, o aspecto valorativo dos itens. No que diz respeito à exceção, o fator extroversão, uma hipótese aqui levantada é que os itens típicos de manejo de impressão enfatizam aspectos próprios da extroversão, como a facilidade nas relações sociais e a externalização de afetos positivos (Bäckström \& Björklund, 2013; Fielden, Kim \& MacCann, 2015). Se isso estiver correto, a presença da variância descritiva também no inventário de dese- 


\section{H. INTERACÃO EM F PSICOLOGIA}

jabilidade social pode ter mantido a correlação positiva com o fator extroversão mesmo após neutralizar os itens. No entanto, essa intuição precisa ainda de um teste empírico mais aprofundado em estudos futuros.

De maneira geral, vale mencionar que a medida externa de desejabilidade social utilizada não pode ser considerada "pura". A literatura mostra que instrumentos psicométricos de autorrelato de desejabilidade social captam não apenas estilo de resposta, mas também traços legítimos de personalidade (Uziel, 2010). Assim, talvez os coeficientes não reflitam, com precisão, o decréscimo na variância valorativa nos itens. Ainda, é possível que parte da redução das correlações após a neutralização se deva à retirada, não intencional, de uma pequena porção de variância descritiva. Novas investigações, portanto, devem buscar estabelecer medidas externas mais confiáveis de desejabilidade social, afım de precisar melhor a associação com os itens neutralizados aqui apresentados.

A estrutura fatorial encontrada também favoreceu a neutralização aqui proposta como um método viável de prevenção de RSD a inventários de personalidade. Ainda que tenha sido necessário selecionar os melhores itens neutralizados no caso do BFI (20 itens), as soluções fatoriais de ambos os instrumentos se mostraram interpretáveis de uma maneira similar àquela típica das versões originais. Um resultado específico dessa análise também chama a atenção. As correlações entre fatores do BFI se mostraram mais atenuadas na versão neutralizada, em comparação à versão original. Estudos anteriores (Bäckström, 2007, Bäckström \& Björklund, 2013, 2016; Bäckström, Björklund \& Larsson, 2009) sugerem que as correlações entre os Cinco Grandes Fatores (e mesmo os fatores de segunda ordem estimados a partir dessas correlações; Bäckström, 2007, Bäckström \& Björklund, 2016) são, em geral, o produto da presença de variância valorativa comum aos itens do instrumento. Assim, as correlações próximas a zero na versão neutralizada do BFI constituem uma evidência adicional de que os procedimentos promoveram a redução da variância valorativa nos itens.

Ainda outro aspecto merece um destaque. A consistência interna das versões neutralizadas dos itens foi ligeiramente inferior àquela dos itens originais. No entanto, o mesmo padrão foi encontrado por Bäckström \& Björklund, (2013) que obtiveram consistência interna menor na versão neutralizada ( 0,73 versus 0,86 para extroversão, 0,70 versus 0,52 para abertura, 0,84 versus 0,81 para conscienciosidade, 0,88 versus 0,70 para socialização e 0,88 versus 0,85 para neuroticismo). Apesar de os instrumentos no estudo citado terem sido diferentes dos usados na atual pesquisa (além disso, continham muito mais itens), o foco é o padrão de diminuição da consistência interna em versões neutralizadas de instrumentos de personalidade. Isso é esperado dada a redução na variância comum entre os itens, decorrente de remover variância valorativa irrelevante.

\section{CONSIDERAÇÕES FINAIS}

De maneira geral, os achados se somam à literatura que sugere ser possível neutralizar itens de autorrelato de personalidade. 0 método da neutralização tem a vantagem de ser simples, prático e acessível a pesquisadores. 0 controle do aspecto valorativo dos itens permite que seja feita, possivelmente, uma avaliação mais acurada do traço de interesse, uma vez que reduz a variância irrelevante nos escores, ordenando de maneira mais fidedigna os indivíduos no contínuo do traço latente.

0 método de neutralização não é uma cura mágica. Ele apenas propõe um olhar mais cuidadoso por parte dos pesquisadores quando estão construindo itens, uma vez que a escrita de um item irá influenciar na forma como as pessoas responderão a ele. Este estudo fez uso da neutralização em um instrumento que avalia os cinco grandes fatores da personalidade e outro que avalia modéstia, mostrando a simplicidade e eficácia do método. Há bons motivos para crer que o procedimento pode ser aplicado, com sucesso, a itens que avaliam muitos outros tipos de traços de personalidade.

\section{CONTRIBUIÇÃO DE CADA AUTOR}

A.C. e N.H. foram responsáveis pela conceitualização, investigação e visualização, pela redação da revisão, edição e rascunho; A.C. pela obtenção de financiamento; e N.H. supervisão.

\section{DECLARAÇÃO DE CONFLITOS DE INTERESSES}

Os autores declaram que não há conflitos de interesses no presente artigo.

\section{FINANCIAMENTOS}

A pesquisa relatada no manuscrito foi financiada parcialmente pela bolsa de mestrado da primeira autora (CNPq, prot. 131700/2016-2)

\section{REFERÊNCIAS}

American Educational Research Association (AERA), American Psychological Association (APA), National Council on Measurement in Education (NCME), Joint Committee on Standards for Educational (JCSE), \& P. T. (US). (2014). Standards for educational and psychological testing (5th ed.). Washington, DC: Amer Educational Research Assn. 


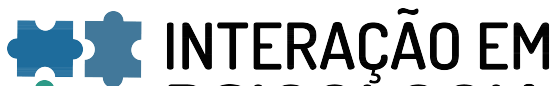 ET PSICOLOGIA}

Bäckström, M. (2007). Higher-order factors in a five-factor personality inventory and its relation to social desirability. European Journal of Psychological Assessment, 23(2), 6370. http://dx.doi.org/10.1027/1015-5759.23.2.63

Bäckström, M., \& Björklund, F. (2013). Social desirability in personality inventories: Symptoms, diagnosis and prescribed cure. Scandinavian Journal of Psychology, 54(2), 1529. http://dx.doi.org/10.1111/sjop.12015

Bäckström, M., \& Björklund, F. (2014). Social desirability in personality inventories: The nature of the evaluative factor. Journal of Individual Differences, 35(3), 144-157. http://doi.org/10.1027/1614-0001/a000138

Bäckström, M., \& Björklund, F. (2016). Is the general factor of personality based on evaluative responding? Experimental manipulation of item-popularity in personality inventories. Personality and Individual Differences, 96, 31-35. http://dx.doi.org/10.1016/j.paid.2016.02.058

Bäckström, M., Björklund, F., \& Larsson, M. R. (2009). Five-factor inventories have a major general factor related to social desirability which can be reduced by framing items neutrally. Journal of Research in Personality, 43(3), 335344. http://dx.doi.org/10.1016/j.jrp.2008.12.013

Bäckström, M., Björklund, F., \& Larsson, M. R. (2012). Social desirability in personality assessment. Em: M. Ziegler, C. MacCann, \& R. D. Roberts (Eds.). New Perspectives on Faking in Personality Assessment (pp. 201-213). New York, NY: Oxford University Press, Inc.

Bäckström, M., Björklund, F., \& Larsson, M. R. (2014). Criterion validity is maintained when items are evaluatively neutralized: Evidence from a full-scale five-factor model inventory. European Journal of Personality, 28(6), 620-633. http://dx.doi.org/10.1002/per.1960

Benet-Martinez, V., \& John, O. E. (1998). Los cinco grandes across cultures and ethnic groups: Multitrait multimethod analyses of the big five in spanish and english. Journal of Personality. 75(3), 729-750.

http://dx.doi.org/10.1037/0022-3514.75.3.729

Borsa, J. C., Damásio, B. F., \& Bandeira, D. R. (2012). Crosscultural adaptation and validation of psychological Instruments. Paidéia, 22(53), 423-432. http://dx.doi.org/10.1590/S0103-863X2012000300014

Fielden, C., Kim, L. E., \& MacCann, C. (2015). Extraversion. International Encyclopedia of the Social \& Behavioral Sciences, 8(1916), 623-627. http://dx.doi.org/10.1016/B978-0-08-097086-8.25099-X

Goldberg, L. R., Johnson, J. A., Eber, H. W., Hogan, R., Ashton, M. C., Cloninger, C. R., \& Gough, H. C. (2006). The international personality item pool and the future of public-domain personality measures. Journal of Research in Personality, 40, 84-96. https://doi.org/10.1016/j.jrp.2005.08.007

Jackson, D. N. (1984). Personality Research Manual (3rd ed.). Port Huron, MI: Research Psychologists Press.
Messick, S. (1995). Validity of psychological assessement. American Psychologist, 50(9), 741-749. http://dx.doi.org/10.1037//0003-066X.50.9.741

Paulhus, D. L. (1988). Balanced inventory of desirable responding (BIDR). Acceptance and Commitment Therapy. Measures Package. http://dx.doi.org/10.1037/0022

Paulhus, D. L. (1991). Measurement and control of response bias. Em:J. P. Robinson, P. R. Shaver, \& L. S. Wrighsman (Eds.), Measures of Personality and Social Psychological Attitudes (pp. 17-59). San Diego, CA: Academic Press.

Paulhus, D. L. (2002). Socially desirable responding: the evolution of a construct. Em:H. I. Brown, D. N. Jackson, \& D. E. Wiley (Eds.), The Role of Constructs in Psychological and Educational Measurement (pp. 49-69). Mahuah, NJ: Erlbaum.

Paulhus, D. L., \& Reid, D. B. (1991). Enhancement and denial in socially desirable responding. Journal of Personality and Social Psychology, 60(2), 307-317. http://dx.doi.org/10.1037/0022-3514.60.2.307

Paulhus, D. L., \& Vazire, S. (2007). The self-report method. Em:R. W. Robins, R. C. Fraley, \& R. F. Krueger (Eds.), Handbook of Research Methods in Personality Psychology (pp. 224-239). New York, NY: The Guilford Press.

Peabody, D. (1967). Trait interferences: evaluative and descriptive aspects. Journal of Personality and Social Psychology, 7(4, Pt.2), 1-18. http://dx.doi.org/10.1037/h0025230

Revelle, W. (2014). Psych: Procedures for personality and psychological research. $\mathrm{R}$ package version 1.4.3. CRAN Project.

Santos, D., \& Primi, R. (2014). Desenvolvimento socioemocional e aprendizado escolar: Uma proposta de mensuração para apoiar políticas públicas. São Paulo: Instituto Ayrton Senna.

Uziel, L. (2010). Rethinking social desirability scales: From impression management to interpersonally oriented self-ontrol. Perspectives on Psychological Science, 5(3), 243-62. http://dx.doi.org/10.1177/1745691610369465

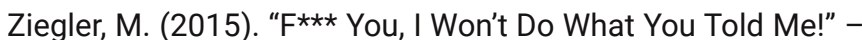
Response biases as threats to psychological assessment. European Journal of Psychological Assessment, 31(3), 153158. http://dx.doi.org/10.1027/1015-5759/a000292

Recebido em: 05/06/2017 Primeira decisão editorial em: 07/08/2017 Aceito em: 22/09/2017 